Chirurg 2019 $\cdot 90: 760$

https://doi.org/10.1007/s00104-019-01014-8

Online publiziert: 14. August 2019

(c) Springer Medizin Verlag GmbH, ein Teil von Springer Nature 2019

\section{Originalpublikation}

Notarnicola M, Felli E, Roselli S et al (2019) Laparoscopic liver resection in elderly patients: a systematic review and meta-analysis. Surg Endosc. https://doi.org/10.1007/s00464019-06840-9

Einführung. Die laparoskopische Chirurgie hat sich gegenüber dem offenen Vorgehen in der Behandlung verschiedener Entitäten in der Viszeralchirurgie durch eine Verringerung postoperativer Komplikationen, Verkürzung des Krankenhausaufenthaltes und ein vergleichbares Gesamtüberleben bzw. Rate an R0-Resektionen als gleichwertig oder gar überlegen erwiesen. Dies wird auch zunehmend für Leberteilresektionen geprüft.

Eine Herausforderung für die Chirurgie ist die Zunahme älterer Patienten und deren erfolgreiche Behandlung. In dieser Metaanalyse wurde die Datenlage für laparoskopische Leberteilresektionen bei älteren Menschen betrachtet.

Methodik. Betrachtet wurden Arbeiten, die bei älteren Patienten offene (OLR) mit laparoskopischen Leberresektionen (LLR) verglichen sowie die Unterschiede der Ergebnisse der laparoskopischen Leberchirurgie bei älteren und nicht älteren Patienten beleuchten. Nach Selektion aus insgesamt 8400 Ergebnissen auf MEDLINE, PubMed, EMBASE und Scopus unter Verwendung der PRISMA-Checkliste wurden fünf Studien zu LLR vergleichend mit OLR bei älteren (Gruppe 1) und vier über LLR bei älteren und nicht älteren Patienten (Gruppe 2) eingeschlossen.

\title{
Laparoskopische Leberresektion bei älteren Patienten
}

Ergebnisse. Es wurden aus neun Studien Ergebnisse von 497 älteren Patienten, welche einer LLR unterzogen wurden, betrachtet. Als älterer Patient definierten sechs Arbeiten ein Alter ab 70 und drei ein Alter ab 75 Jahren. In beiden Gruppen war die Pathologie heterogen, wobei das Gros von kolorektalen Lebermetastasen eingenommen wurde, gefolgt vom hepatozellulären Karzinom und dem cholangiozellulären Karzinom. Als primäre Endpunkte wurden intraoperativer Blutverlust, Transfusionspflichtigkeit, postoperatives Leberversagen und Mortalität, das relative Risiko (RR) für eine Komplikation nach ClavienDindo III/IV sowie ein postoperatives Galleleck definiert. Die Länge des Krankenhausaufenthaltes, Operationsdauer und R0-Resektion waren sekundäre Endpunkte.

In Gruppe 1 wurden 640 mit OLR und 385 Patienten mit LLR behandelt, zu einer Konversion von $6 \%$ kam es nur in einer Arbeit, welche Majorresektionen einschloss (übrige $0 \%$ ). Die postoperative Mortalität zeigte sich in beiden Altersgruppen als gering. Intraoperativer Blutverlust (LLR vs. OLR $-240 \mathrm{ml}$ ), Transfusionspflichtigkeit (8\% LLR vs. $13,1 \%$ OLR), postoperatives Leberversagen (18,3\% LLR vs. $28,4 \%$ OLR), Clavien-Dindo III/IV (RR 0,48 zugunsten der LLR) und ein postoperatives Galleleck (2,5\% LLR vs. 3,2\% OLR) sowie Krankenhausaufenthalt $(-4,93$ Tage bei LLR), Operationszeit ( $-23 \mathrm{~min}$ bei LLR) und R0-Resektion (91,1\% LLR vs. $88,4 \%$ OLR) fielen zugunsten der LLR aus.

Gruppe 2 schloss 112 ältere ( 276 nicht ältere Patienten ein. Postoperatives Leberversagen trat mit einem $R R$ von 0,65 bei ÄP auf, ein Clavien-Din- do III/IV war bei ÄP weniger häufig (RR $1,47)$, ein Galleleck trat ohne signifikanten Unterschied auf. Der Krankenhausaufenthalt hatte einen medianen Unterschied von 0,72 Tagen, die Operationszeit war bei ÄP kürzer (-18 min), die R0-Resektion gelang ohne signifikanten Unterschied.

Fazit. Die hier vorgestellte Arbeit untersucht die Ergebnisse einer laparoskopischen Leberresektion bei älteren Menschen. Es wird gezeigt, dass ein minimal-invasives Vorgehen bei diesen $\mathrm{Pa}$ tienten realisierbar und sicher ist. Typische operationsassoziierte Komplikationen sowie das onkologische Ergebnis waren vergleichbar. Es konnte kein signifikanter Unterschied an Komplikationen oder Blutverlust und -transfusionen verzeichnet werden, womit anzunehmen ist, dass ältere Patienten von einem minimalinvasiven Vorgehen auch unter Berücksichtigung ihrer Komorbiditäten profitieren können.

\section{Korrespondenzadresse}

PD Dr. Dr. M. Ardelt

Klinik für Allgemein-, Viszeral- und Gefäßchirurgie, Universitätsklinikum Jena Am Klinikum 1, 07747 Jena, Deutschland Michael.Ardelt@med.uni-jena.de

Interessenkonflikt. R. Keiner, M. Ardelt und U. Settmachergeben an, dass kein Interessenkonflikt besteht. 